\title{
A model for segmentation of CT images of liver lesions based on regional fitting and gradient information
}

\section{Baoshan Xue}

Soochow University https://orcid.org/0000-0002-0341-815X

Fengfeng Zhang ( $\square$ zhangfengfeng@suda.edu.cn )

Soochow University https://orcid.org/0000-0001-5250-9421

\section{Xiaojian Yan}

Soochow University

\section{Rongmiao Wang}

Soochow University

Research article

Keywords: Tumor segmentation, Gradient information, Region fitting, Segmentation model, Active contour, Liver

Posted Date: October 21st, 2020

DOI: https://doi.org/10.21203/rs.3.rs-92780/v1

License: (c) (i) This work is licensed under a Creative Commons Attribution 4.0 International License. Read Full License 


\title{
A model for segmentation of CT images of liver lesions based on regional
}

\section{fitting and gradient information}

\author{
Baoshan Xue ${ }^{1}$, Fengfeng Zhang ${ }^{1,2}$, Xiaojian Yan ${ }^{1}$, Rongmiao Wang ${ }^{1}$ \\ 1. School of Mechanical and Electrical Engineering, Soochow University, Suzhou, 215006, China; \\ 2. Collaborative Innovation Center of Suzhou Nano Science and Technology, Soochow University, Suzhou, 215123, China
}

* Corresponding Author: Fengfeng Zhang; zhangfengfeng@ suda.edu.cn; Tel.: +86-186-0512-7012

\begin{abstract}
:
Background: The Computerized tomography (CT) images of liver have such disadvantages as uneven gray scale, fuzzy boundary and missing, so the commonly used image segmentation model of liver lesions has low segmentation accuracy.

Methods: We propose a new hybrid active contour model based on regional fitting and gradient information for segmenting CT images of liver lesions. Firstly, the problem of uneven gray scale of liver lesions image was solved by local area fitting method, and the gradient information of liver lesions image was integrated to enhance the detection ability of the model on the edge of liver lesions. Secondly, we introduce the region area term, which can keep the image segmentation curve smooth in the process of segmentation, and effectively control the direction and speed of curve evolution. Finally, the performance of the Distance Regularized Level Set Evolution (DRLSE) model, Region-Scalable Fitting (RSF) model and the present model was compared in the segmentation of liver lesions.
\end{abstract}

Results: It can be concluded from the experimental results that: compared with DRLSE model and RSF model, the average Dice similarity coefficient reached $97.7 \%$, ncreased by $12.7 \%$ and $11.7 \%$ respectively; the under segmentation rate was $2 \%, 9 \%$ and $17 \%$ lower, and the over segmentation rate was $1.6 \%$.

Conclusion: Therefore, the segmentation model proposed in this paper has excellent segmentation performance and greatly improves the segmentation accuracy of liver lesions.

Keywords:

Tumor segmentation; Gradient information; Region fitting; Segmentation model; Active contour; Liver

\section{Background}

The development of medical image segmentation technology ${ }^{[1,2]}$ has passed the stage of manual segmentation, and has entered the stage of semi-automatic and full-automatic segmentation ${ }^{[3,4,5,6,7,8]}$. However, when contrast agent flows into the hepatic artery, the image information in the tumor area is unevenly enhanced, resulting in blurry boundary, partial missing and uneven gray scale in CT images, so there will be a large error in the segmentation results of liver lesions. The commonly used medical image segmentation models of liver ${ }^{[9]}$ can be divided into several categories according to its principle, such as region-based feature method, active contour model method, statistical shape method, clustering method and graph theory method. Where the active contour model method, due to its simple and efficient computing performance, and can well extract the deformation contour and other advantages, it is widely used in the medical image processing of liver with complex features. The active contour model method has developed rapidly since it was proposed. Caselles et al. ${ }^{[10]}$ proposed the geodesic active contour model (GAC model), which abandoned the dependence of Snake model ${ }^{[11]}$ on parameters and added the level set, making the contour curve closer to the topological structure of the target object. However, the results of CT images of liver lesions with blurred boundaries, partial missing and uneven gray scales are not satisfactory. $\mathrm{Li}$ et al. ${ }^{[12]}$ proposed a level set model (DRLSE model) for distance regularization based on GAC model, which improved the evolution speed of segmentation curve. Zhang ${ }^{[13]}$, Wang ${ }^{[14]}$ et al. constrained the evolution of active contour by introducing local information, to some extent, it 
improves the ability of processing CT images of the liver. Wei et al. ${ }^{[15]}$ defined the edge stop function and weight coefficient, so as to improve the model's ability of extracting liver boundary information. However, these methods still can not achieve the cross region jump evolution of segmentation curve, and can not complete the task of segmentation of liver lesions with fuzzy boundary or chaotic gradient information. Chan et al. ${ }^{[16]}$ proposed a region-based level set method (Chan-Vese model,CV model), it has a good effect on the segmentation of images with significantly different pixel values between the foreground and the background of liver focus, this model has poor ability of segmentation for CT images of liver with complex features such as blurred boundary, partial absence and uneven gray scale. Li et al. ${ }^{[17]}$ proposed an active contour model (RSF model) based on fitting energy of contractible region. However, it is easy to fall into the local minimum when solving the energy functional, which is more suitable for global segmentation tasks. Akram et al. ${ }^{[18]}$ proposed a locally and globally fitted image segmentation model. $\mathrm{ZHANG}^{[19]}$, ZHAO ${ }^{[20]}$ et al., and Soomro et al. ${ }^{[21]}$ proposed a region-based active contour model weighted by edge stop function. Although these models can process CT images of liver with complex features such as gray inequality, but it leads to a decrease in the evolutionary stability of the segmentation curve, it is also difficult to complete the task of segmenting the local area of the liver.

In recent years, the mainstream deep learning algorithm convolutional neural network $^{[22]}(\mathrm{CNN})$ has made great progress in medical image segmentation and other tasks. In the final analysis, the methods based on convolutional neural network are to establish the mapping of pixels and pixels in a certain range to instances or categories through known sample data. However, the mechanical mapping model has obvious defects, almost no migration ability and generalization ability, and due to the complexity of the mapping model, slight changes in the input data will lead to unexpected errors. In addition, medical images have the characteristics of less semantic content, low imaging quality, less data ${ }^{[23]}$, and multiple modes ${ }^{[24]}$. In 2016, Ben Cohen et al. ${ }^{[25]}$ used fully convolutional network (FCN) for the first time to solve the problem of liver and liver tumor segmentation. U-net [26] took FCN as the backbone to fully mine the multi-scale features of the image. In the segmentation process, the above neural network realizes the automatic segmentation of liver and liver tumor through the independent classification of pixels. However, on the one hand, it is still urgent to solve the problem of too few data samples, and the segmentation labels in the training data need to be manually made by professional doctors, which is costly; on the other hand, other pixel categories in the neighborhood are not used in the classification process of each pixel Information is easy to appear small target area missing segmentation and fuzzy target boundary segmentation.

Based on the above situation, this paper proposes a model for segmentation of CT images of liver focus based on regional fitting and gradient information. It solves the problem that the common segmentation model of liver lesions image has a low accuracy in the segmentation of liver CT image with uneven gray scale, fuzzy boundary or absence. By combining local area fitting of liver lesions with image gradient information, it not only overcomes the uneven image gray level, but also enhances the edge detection ability of the model. And then by introducing the area term, so that the segmentation curve in the evolution process to keep smooth, and effectively control the evolution direction and speed. These processing can effectively improve the accuracy of liver focus CT image segmentation, so that the model in this paper has excellent performance of liver focus segmentation.

\section{Methods}

\section{Boundary based active contour model}

The boundary-based active contour model controls the movement speed of the curve through the boundary information of the liver image. The Geodesic Active Model (GAC) proposed by Caselles and Kimmel et al. ${ }^{[10]}$ is one of the most classical boundary-based Active contour models. Then $\mathrm{Li}$ et al. ${ }^{[19]}$ proposed a level set model (DRLSE) for distance regularization based on GAC model. By introducing a distance regularization term to keep the evolving surface regular, the distance regularization term is introduced to keep the evolving surface regular and reduce the reinitialization step in the iterative process, thus the evolution of curve for segmenting liver focus is accelerated. The GAC model and its subsequent development are often applied to liver segmentation tasks ${ }^{[12,27,28,29]}$. These models, in the 
process of liver segmentation, define an edge detection equation. At the edge of the contour where the information gradient of the liver focus is large, the value of the equation is close to 0 , the force driving the evolution of the segmentation curve is small, and the curve will stop at the contour of the liver focus; at the location where the gray scale of the liver focus area is similar, the image gradient is small, so that the value of the equation is close to 1 , there will be a large external force driving the segmentation curve to move to the location where the information gradient of the image is large.

For the boundary based active contour model, the segmentation curve depends on the size of the gradient information of the image, and can only move in the direction where the gradient information of the image increases. In the segmentation task of liver lesions, when an image with blurred boundary or gradient information features is encountered, the performance of segmentation will decrease, and this model can not realize the evolution of cross-region segmentation, which is only suitable for local segmentation tasks.

\section{Area based active contour model}

The borderless active contour model proposed by Chan and Vese ${ }^{[16]}$ is the most classical model based on the segmentation of CT images of the liver in the region, known as the Chan-Vese model. In various tasks related to liver segmentation ${ }^{[30,31]}$, The $\mathrm{CV}$ model uses an arbitrary curve $\mathrm{C}$ to divide the images of liver focus into two parts: the inside part and the outside part. Then the energy function is defined and minimized. When the value of the energy function is minimum, the image is divided into foreground and background.

Later, Li et al ${ }^{[17]}$ proposed an active contour model (RSF) based on the fitting energy of contractible region. On the basis of local fitting, the model can effectively deal with the problem of uneven gray scale of liver lesions by fitting energy statistics of global pixel points. But because only the local region information is calculated, the model is easy to fall into the local minimum value when solving the energy function.

From the point of view of the composition of the energy function, it can be seen that both $\mathrm{CV}$ model and RSF model are segmentation models based on global statistics. They are generally only suitable for global segmentation, and cannot be directly applied to such local segmentation tasks as liver lesions.

\section{Segmentation model of liver focus image based on region fitting and gradient information}

We propose a hybrid active contour model based on region fitting and gradient information, which can well solve the shortcomings of the above models, and has excellent segmentation ability in the face of the CT image of liver lesions with blurred boundary, partial missing and uneven gray level. We define the energy functional of the segmentation model as formula (1):

$$
E(C)=\beta F i t(C)+\lambda \operatorname{Grad}(C)
$$

In the formula, $\mathrm{C}$ represents any curve, which divides the CT image of liver focus into curve inside and curve outside. $\operatorname{Fit}(C)$ is the local area fitting term of liver CT image, $\operatorname{Grad}(C)$ is the gradient information item of liver image. Different from the RSF model, the local area fitting term $F i t(C)$ only calculates the sum of the fitting values of all points on the segmentation curve $\mathrm{C}$ of liver lesions. The complete formula of local area fitting term is defined as formula (2):

$$
\operatorname{Fit}(C)=\int_{0}^{L(C)} \varepsilon_{x}^{F i t}\left(C, u_{x}, v_{x} d s\right)
$$

In this model, the gradient information item $\operatorname{Grad}(C)$ of liver focus CT image is added, that is, the gradient energy of the pixels on the segmentation curve $\mathrm{C}$ is calculated by the liver focus boundary detection equation $g$, it overcomes the problem of easily falling into local minimum value when solving energy functional. The formula for defining the gradient information item is as follows (3):

$$
\operatorname{Grad}(C)=\int_{0}^{L(C)} g(\nabla u \cdot C) d s
$$

Because the segmentation curve $\mathrm{C}$ of liver lesions is unknown, it is difficult to solve the evolution equation directly. According to the thought of level set ${ }^{[32]}$, We use the level set function $\phi$ to replace the arbitrary partition curve $\mathrm{C}$, and the evolution of the plane curve is also replaced by the evolution of the three-dimensional surface. Then, the points on the three-dimensional surface and the points on the surface plane are mapped by introducing the heviside function $H(\phi)$. Finally, Dirac function $\delta(\phi)$ is added to obtain the set of points on the zero horizontal plane of the curved surface. Thus, the energy functional can be expressed as formula (4) and (5): 


$$
\begin{aligned}
& \operatorname{Fit}(C)=\int_{\Omega_{x}} \delta \phi(x) \int_{\Omega_{y}} M(x, y)\left(H \phi(y)\left(I(y)-u_{x}\right)^{2}+\right. \\
& \left.(1-H \phi(y))\left(I(y)-v_{x}\right)^{2}\right) d y d x \\
& \operatorname{Grad}(C)=\int_{\Omega_{x}} g \delta \phi(x)|\nabla \phi| d x
\end{aligned}
$$

In order to keep the surface $\phi$ stable as it evolves, it is necessary to reinitialize the level set surface $\phi$ before each iteration, but it has also led to a large increase in the amount of computation. By adding distance regularization items $R_{p}$ into the model in this paper, the regularization term $R_{p}$ calculates the sum of the corresponding energy density values at the zero level set, so that the gradient of the three-dimensional surface is always 1 during the evolution process, that is, the value of the energy density function is minimum, so as to realize the acceleration of the evolution of the surface. The definition formula is as follows (6):

$$
\mathrm{R}_{p}=\int_{\Omega_{x}} p(|\nabla \phi(x)|) d x
$$

In order to keep the segmentation curve of liver focus smooth in the evolution process, we add the regional area term in this model as formula (7):

$$
A=\int_{\Omega_{x}} g H \phi(x) d x
$$

In the area term, the detection equation $g$ of the boundary of liver lesions is used as the dynamic coefficient, which can accelerate the evolution of the segmentation curve in the homogeneous region of liver lesions. However, in regions with a larger gradient, a smaller $g$ value will slow down the evolution of the segmentation curve. Since we also defined the level set surface $\phi$, when the initial curve is defined outside the contour of the liver focus, the segmentation curve should be shrunk inward, that is, the area term coefficient should be less than zero. Inside the contour of the liver focus, the opposite is true. Therefore, the area term also has the effect of controlling the direction and speed of the movement of the segmentation curve.

In the actual segmentation of liver focus, the values on the three-dimensional surface function $\phi$ are distributed discretely, so it is difficult to find the exact zero horizontal plane. In order to improve the robustness of the segmentation model of CT images of liver lesions, the Heaviside matrix and Dirac matrix were replaced with regularized versions in this model. The level set model of this paper is completed. Therefore, the energy functional formula of the segmentation model of liver lesions is shown in equation (8) :

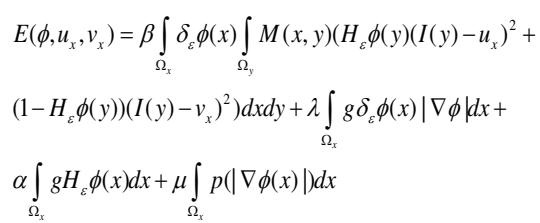

$u_{x}$ and $v_{x}$ calculate the gray mean values inside and outside the segmentation curve over a local region. After obtaining the complete energy functional, we use the gradient descent method to minimize the energy functional. First, $\phi$ is fixed, then the partial derivatives of $u_{x}$ and $v_{x}$ are obtained, and the corresponding Lagrangian equation (9) is constructed:

$$
\left\{\begin{array}{l}
\int M(x, y) H_{\varepsilon} \phi(y)\left(I(y)-u_{x}\right) d y=0 \\
\int M(x, y)\left(1-H_{\varepsilon} \phi(y)\right)\left(I(y)-v_{y}\right) d y=0
\end{array}\right.
$$

Next, we fixed $u_{x}$ and $v_{x}$, then calculated the partial derivative of $\phi$, and finally obtained the evolution equation of the surface of the level set (10) :

$$
\begin{aligned}
& \frac{\partial \phi}{\partial t}=\beta \delta \phi(x) \int_{\Omega_{y}} M(x, y) \delta \phi(y) \cdot\left(\left(I(y)-u_{x}\right)^{2}-\right. \\
& \left.\left(I(y)-v_{x}\right)^{2}\right) d y+\lambda \delta \phi(x) \operatorname{div}\left(g \frac{\nabla \phi(x)}{|\nabla \phi(x)|}\right)+ \\
& \alpha \delta \phi(x)+\mu \operatorname{div}\left(d_{p}(|\nabla \phi(x)| \nabla \phi(x))\right)
\end{aligned}
$$

The flow chart of image segmentation of liver lesions based on the segmentation model of liver lesions proposed in this paper is shown in figure 1 : 


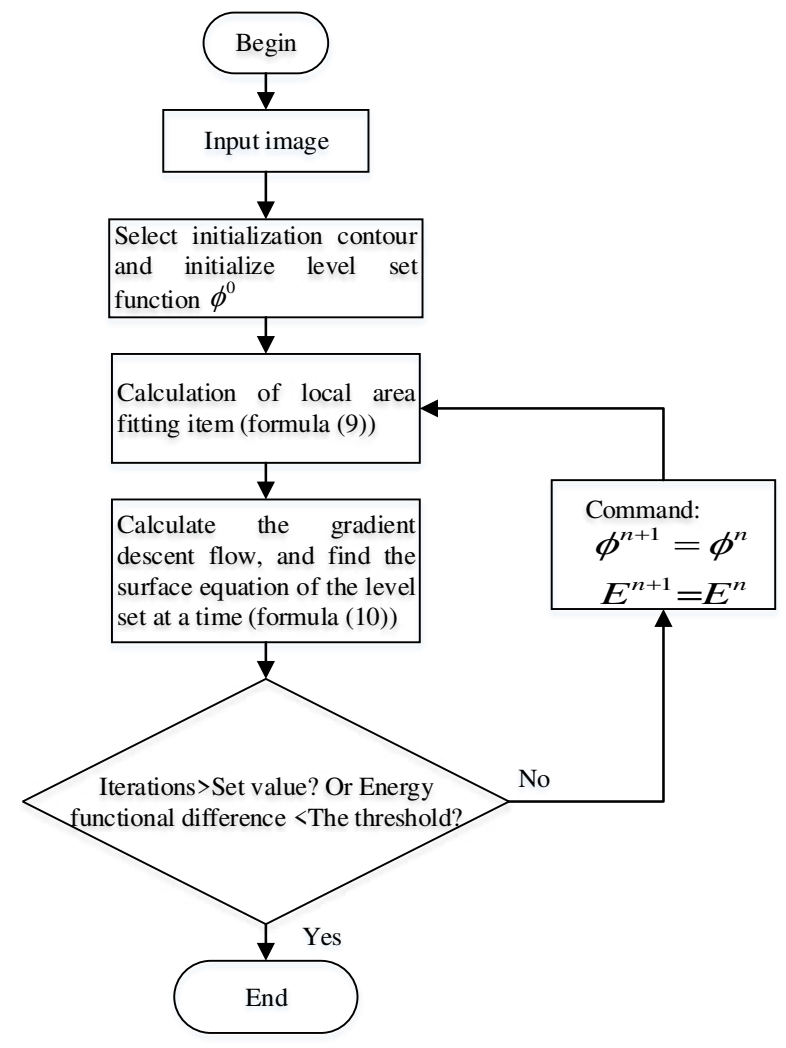

\section{Results}

\section{Experimental materials and parameter Settings}

In this study, the CT images of liver tumors were taken as the experimental objects, and the CT images were obtained from the public challenge dataset of the MICCAI liver tumor segmentation challenge. The experimental environment is Windows10 PC, the processor is inter $(\mathrm{R})$ core $\mathrm{i} 5-3230 \mathrm{~m} 2.6 \mathrm{GHz}$, the memory is $8 \mathrm{~GB}$, through $\mathrm{C}++$ and Matlab2015 hybrid programming. In order to verify the effectiveness of the model in this paper, the DRLSE model, RSF model and the model in this paper were applied to the segmentation task of liver lesions in arterial phase images, and the segmentation performance was compared.

\section{Liver tumor segmentation}

We defined the CT images with clear tumor boundaries as category $\mathrm{A}$, the $\mathrm{CT}$ images with fuzzy tumor boundaries as category $\mathrm{B}$, and the $\mathrm{CT}$ images of large-area tumors as category $\mathrm{C}$, respectively. The CT images of these three types of liver lesions were used for comparative experiments. The parameter settings are as follows: The coefficient of the local area fitting term $\beta=1.5$, Fitting radius $r a d=8$, the coefficient of the image gradient term $\lambda=1.5$, the coefficient of the distance regularization term $\mu=0.2$, the coefficient of the area term $\alpha=3$.

In tumor images with clear boundaries (category A), the area of liver tumor is characterized by uneven gray scale, relatively high brightness compared with liver parenchyma, and clear boundary contour. The box in the figure is the position of the initial contour: $\operatorname{phi}(274: 296,180: 207)=1$. The results of the experiment are shown in figure 2 .

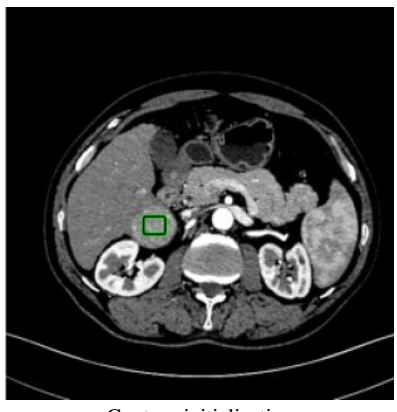

Contour initialization

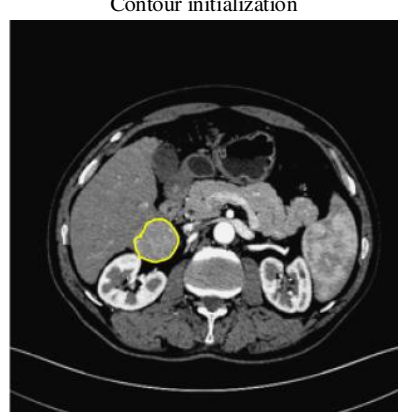

RSF

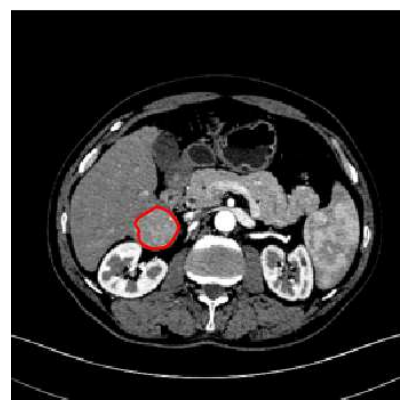

DRLSE

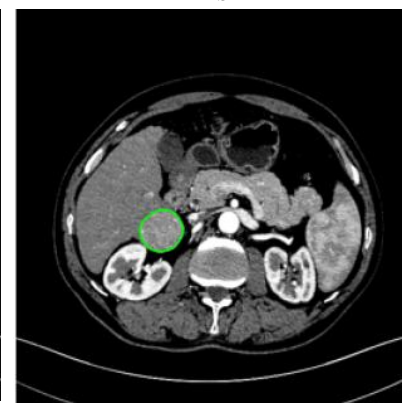

The model of this paper 
As can be seen from the result of segmentation: Both the DRLSE model and the RSF model can perform the task of tumor segmentation well on the whole. However, due to the weak ability of these two segmentation models to process images of liver lesions with uneven gray scale, there is still undersegmentation in local areas. The results obtained by using the segmentation model in this paper can be seen that the tumor region is completely segmented. In addition, the results of segmentation have regular and smooth boundary contour due to the addition of area item in the model.

In tumor images with fuzzy edge features (Category B), there was an obvious uneven gray enhancement in the tumor area, and the gray of the edge was similar to that of the liver parenchyma. The box in the figure is the position of the initial contour: phi(274:296,180:207)=1. The results of experimental are shown in figure 3.

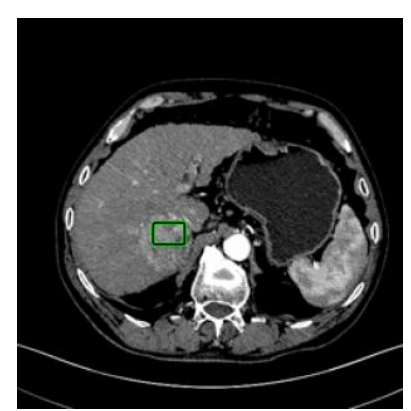

Contour initialization

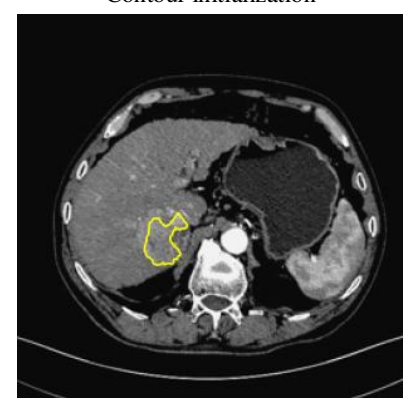

RSF

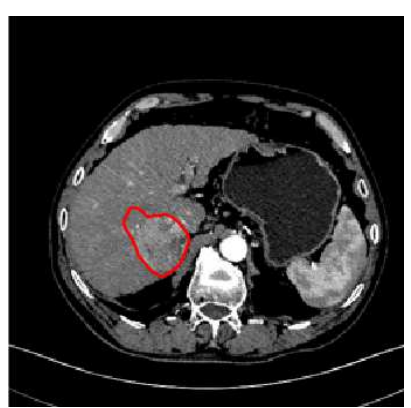

DRLSE

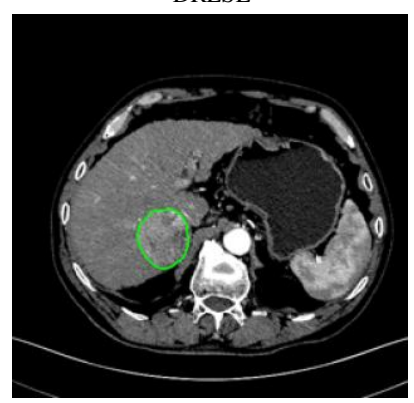

The model of this paper
When the DRLSE model is used to segment the liver focus image with fuzzy boundary, the edge leakage occurs in the upper left position of the tumor, which is caused by too much dependence on the gradient information of the liver lesions image. RSF model is easy to fall into local minimum value when solving energy functional, so it can't be successfully segmented in the upper left and lower right regions of the tumor. The model in this paper fuses the fitting features of local regions and the image gradient features, so that the problem of local minima is avoided, and the ability to distinguish fuzzy boundaries is enhanced. It can be seen that the fuzzy area on the upper left and the uneven gray level area on the lower right have been successfully segmented. Compared with the other two models, the segmentation effect of the model in this paper is better.

In images with a larger tumor area (category $\mathrm{C}$ ), there was uneven enhancement of the gray level of the tumor region, and it was adjacent to the vascular structure. Position of initial contour: phi(269:313,177:213)=1, The results of the experiment are shown in figure 4.

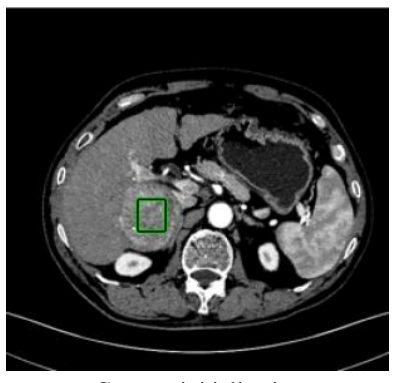

Contour initialization

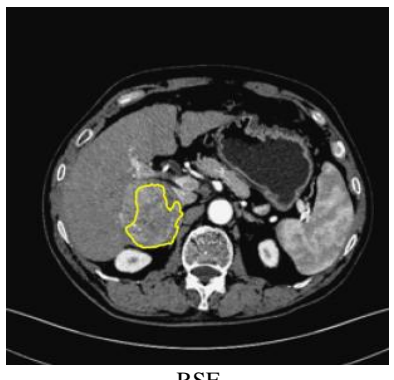

RSF

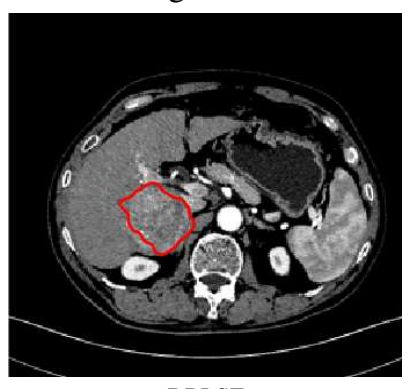

DRLSE

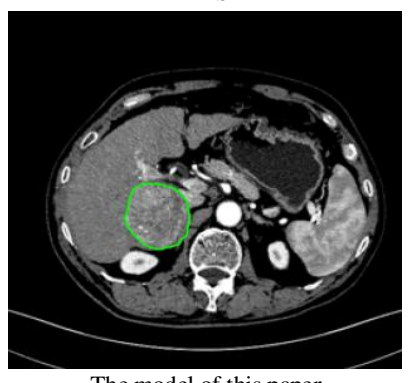

The model of this paper
Since it was difficult to deal with the fuzzy boundary of liver lesions with the DRLSE model, oversegmentation occurred in the upper left region of the tumor and undersegmentation occurred in the lower left region. The results of segmentation of RSF model showed that the evolution curve did not cross the boundary at the edge of the tumor. However, only the local gray fitting feature is considered, which leads to the local minimum value and finally the under segmentation. And the results obtained by using the model in this paper to segment the image, it can be clearly seen that the segmentation curve stops at the edge of the tumor more accurately, and the adjacent sites of the tumor and the blood vessels were also well differentiated.

\section{Energy functional curve}

In the experiment of image segmentation of three kinds of liver tumors mentioned above, we recorded the energy value of the model in this paper after each iteration, and made corresponding energy curves respectively. As shown in figure 5 , the energy curve shows a downward 
trend in the evolution process and finally tends to be stable, that is, the energy of the model reaches the minimum value. In the process of image segmentation, a stable segmentation result can be obtained through the model in this paper.
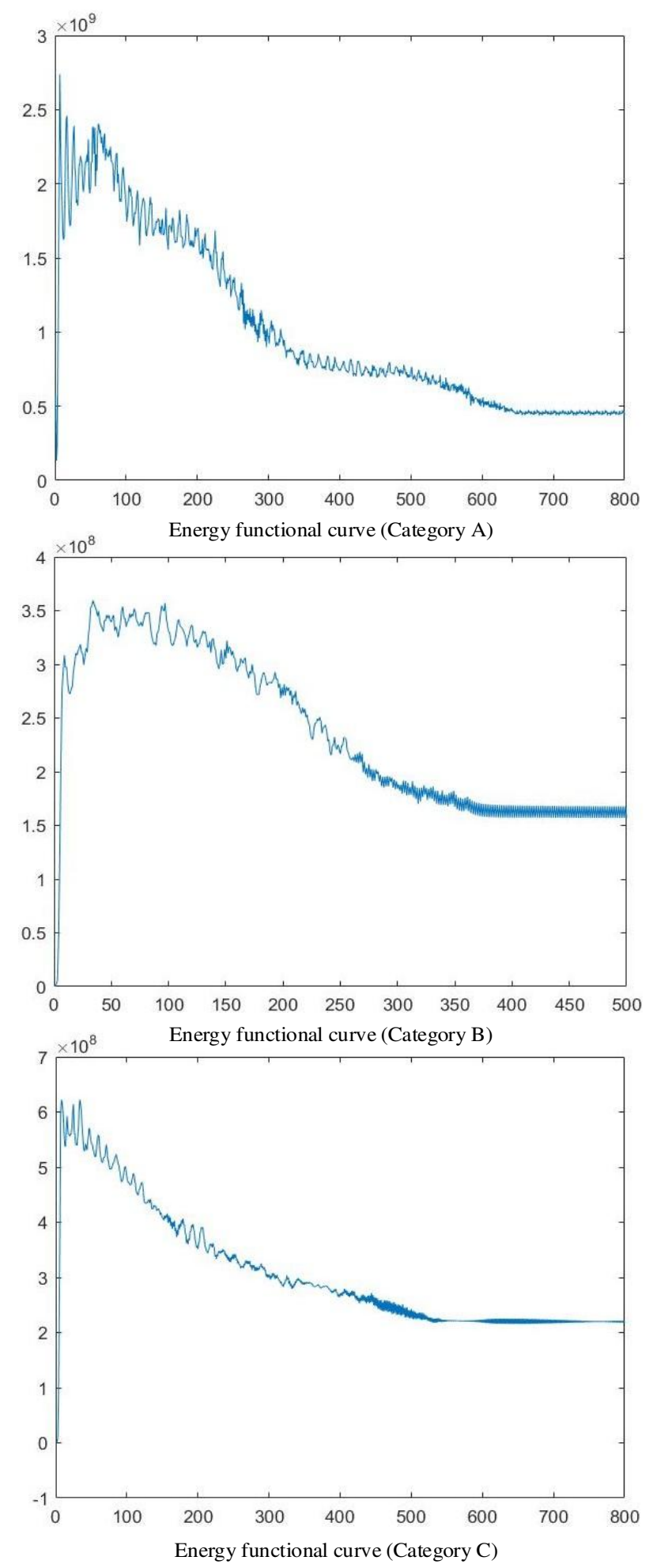

\section{Surface of level set}

We also made the three dimensional surfaces of level set of the model in this paper, as shown in figure 6 . The red curve in the figure represents the zero horizontal plane, that is, the contour of the target boundary obtained after the segmentation is completed. All surfaces are regular and uniform, which shows that the level set surface is always stable in the evolution process, so the model in this paper can get accurate numerical solutions.

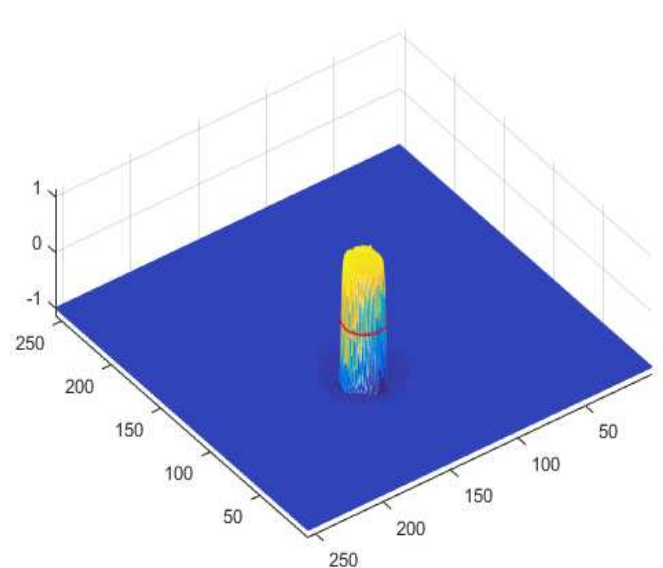

Surface of level set (Category A)

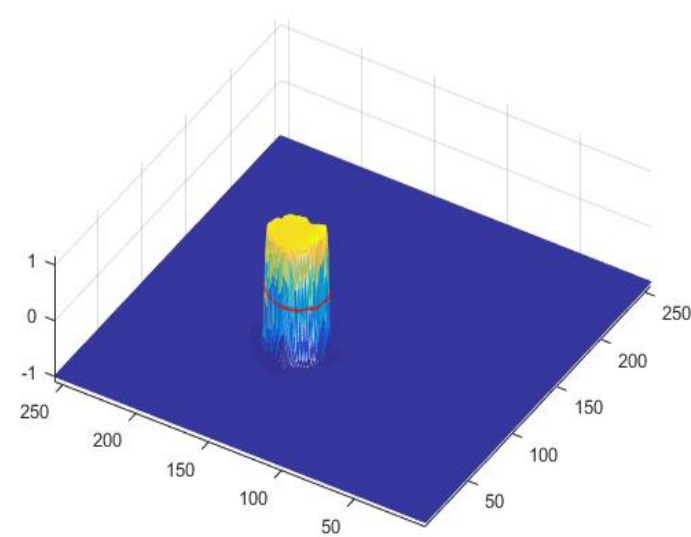

Surface of level set (Category B)

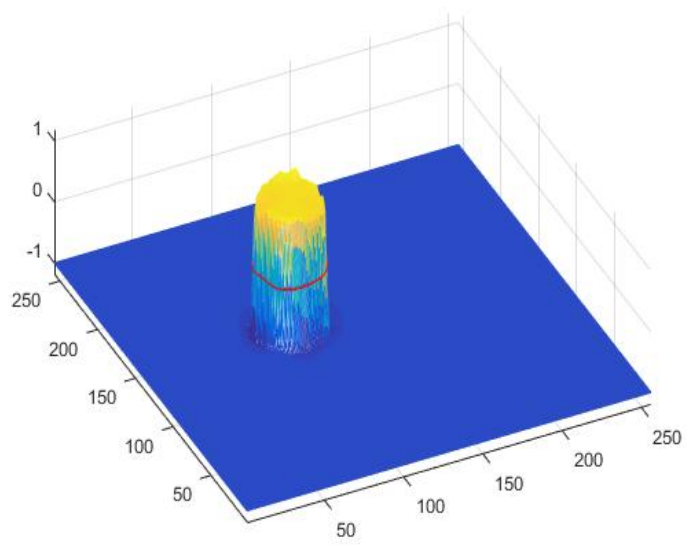

Surface of level set (Category C)

\section{Segmentation of liver nodules}

In this paper, the segmentation experiment of nodules in the liver was carried out. CT images of liver nodules were obtained from the Department of Radiology, the First Affiliated Hospital of Soochow University. The parameters are set as follows: The coefficient of the local 
area fitting term $\beta=1$, Fitting radius $\mathrm{rad}=3$, The coefficient of the image gradient term $\lambda=1$, The coefficient of the distance regularization term $\mu=0.2$, The coefficient of the area term $\alpha=3$. The results of the experiment are shown in figure 7 , the nodule area presents as a low-density mass, and the boundary is also clear relative to the tumor. Therefore, better segmentation results can be obtained by using the model in this paper.
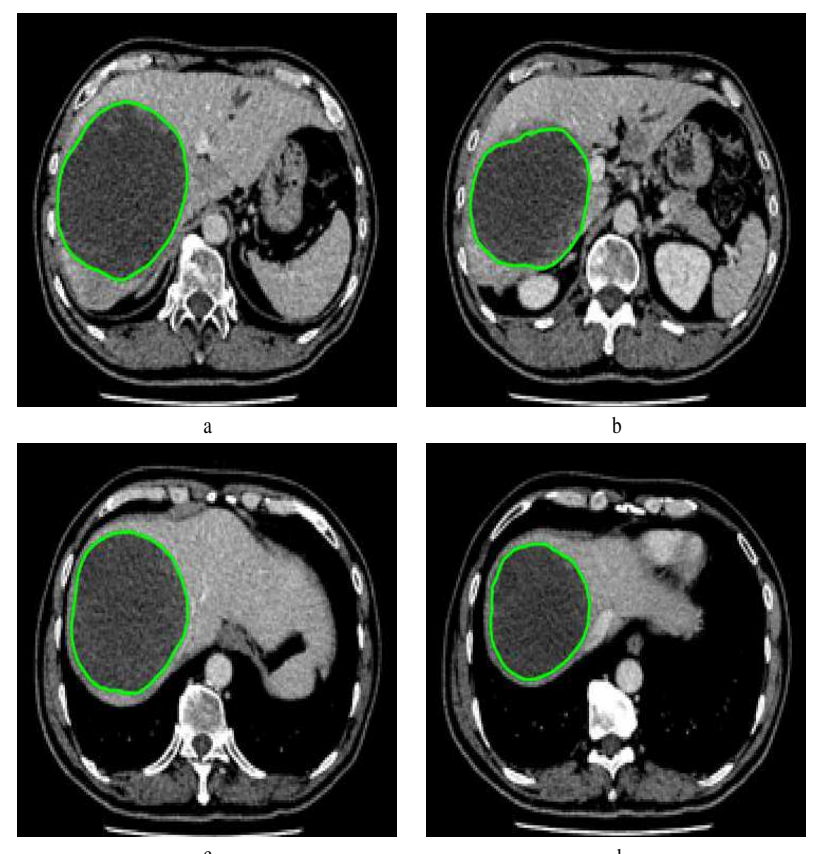

\section{Quantitative evaluation of segmentation results}

In order to quantitatively evaluate the segmentation results, this paper adopts three commonly used evaluation indexes: Dice similarity coefficient(Dice), undersegmentation rate(UR) and oversegmentation $\operatorname{rate}(\mathrm{OR})$. The calculation formulas of Dice, OR and UR are defined as follows:

$$
\begin{aligned}
& \text { Dice }=\frac{2\left|R_{g t} \mathrm{I} R_{\text {seg }}\right|}{\left|R_{g t}\right|+\left|R_{\text {seg }}\right|} \\
& O R=\frac{\left|R_{\text {seg }}\right|-\left|R_{g t} \mathrm{I} R_{\text {seg }}\right|}{R_{g t}+\left|R_{g t} \mathrm{I} R_{\text {seg }}\right|}
\end{aligned}
$$

$$
U R=\frac{\left|R_{g t}\right|-\left|R_{\text {seg }} \mathrm{I} R_{g t}\right|}{R_{g t}+\left|R_{\text {seg }} \mathrm{I} R_{g t}\right|}
$$

Where $R_{g t}$ is the ground truth, $R_{\text {seg }}$ is the segmentation result of the model. We calculated three types of evaluation indicators, and added Iterations and Times as the performance evaluation indicators of segmentation. The results are shown in table 1.

\section{Discussion}

\begin{tabular}{ccccccc}
\hline & Model & Dice & UR & OR & Iterations & Times \\
\hline \multirow{2}{*}{ Category A } & DRLSE & 0.923 & 0.082 & 0.015 & 389 & 6.21 \\
& RSF & 0.938 & 0.096 & 0.011 & 457 & 8.67 \\
& This paper & 0.981 & 0.019 & 0.013 & 611 & 9.02 \\
Category B & DRLSE & 0.802 & 0.094 & 0.193 & 225 & 3.59 \\
& RSF & 0.839 & 0.258 & 0.012 & 358 & 3.97 \\
& This paper & 0.973 & 0.025 & 0.019 & 369 & 4.12 \\
Category C & DRLSE & 0.825 & 0.165 & 0.161 & 514 & 5.35 \\
& RSF & 0.807 & 0.237 & 0.024 & 561 & 6.09 \\
& This paper & 0.978 & 0.022 & 0.016 & 327 & 7.15 \\
& DRLSE & 0.850 & 0.114 & 0.123 & 5.18 \\
\hline \multirow{2}{*}{ Means } & RSF & 0.861 & 0.197 & 0.016 & 535 & 6.60 \\
& This paper & 0.977 & 0.022 & 0.016 & 510 & 6.76 \\
\hline
\end{tabular}

When using the model in this paper to segment tumors, the average Dice similarity coefficient reached $97.7 \%$, the undersegmentation rate was $2 \%$, and the oversegmentation rate was $1.6 \%$. Compared with the other two models, the performance of segmentation has been greatly improved, especially the images of liver 
lesions with complex features are segmented, the model in this paper combines the gradient information and the local gray level fitting information at the same time, and sacrifices the segmentation time properly, which makes the segmentation have high accuracy, low over segmentation rate and under segmentation rate.

\section{Conclusions}

The segmentation accuracy of the liver lesion segmentation model is reduced by the uneven gradient information in the lesion area, the missing edge contour, etc , this paper proposes an active contour model based on region fitting and gradient information for image segmentation of liver lesions. The model in this paper overcomes the problem of uneven image gray level by fitting the local area of liver lesions. At the same time, combining with the gradient information of the images of liver lesions to enhance the edge detection ability of the model and effectively prevent the model from falling into the wrong local minimum value in the evolution process. Thus, CT images of liver lesions with complex features can be segmented more accurately. The experimental results show that: The active contour model is proposed in this paper, in the task of segmenting liver tumors, the average Dice similarity coefficient can reach $97.7 \%$, which is $12.7 \%$ and $11.7 \%$ higher than that of DRLSE model and RSF model, respectively. The average undersegmentation rate was $2 \%$, which was reduced by $9 \%$ and $17 \%$ respectively compared with DRLSE model and RSF model. The oversegmentation rate was $1.6 \%$. Therefore, the model proposed in this paper, which is used to segment the CT images of liver lesions, can obtain higher accuracy and lower undersegmentation rate and oversegmentation rate, the segmentation accuracy of liver lesions was greatly improved.

\section{Abbreviations}

CT: Computerized tomography

DRLSE: Distance Regularized Level Set Evolution

RSF: Region-Scalable Fitting

GAC: Geodesic active contour

CV: Chan-Vese

CNN: Convolutional neural network

FCN: Fully convolutional network

Dice: Dice similarity coefficient

UR: Undersegmentation rate

OR: Oversegmentation rate

\section{Declarations}

Ethics approval and consent to participate: The CT images of liver tumer were obtained from the public challenge dataset of the MICCAI liver tumor segmentation challenge. CT images of liver nodules were obtained from the First Affiliated Hospital of Soochow University, and also approved by the Ethics Committee of the First Affiliated Hospital of Soochow University.

Consent for publication: Not applicable.

Availability of data and materials: Authors agree to make all published data available.

Competing interests: Authors state no conflict of interest.

Funding: National Key Research and Development Project (2018YFB1307700) funded the study .

Authors' contributions: BSX contributed to the drafting and editing of this document. XJY and RMW designed experiments and completed the experimental data acquisition. FFZ guided the thinking. All authors read and approved the final manuscript.

Acknowledgements: The authors extend their gratitude to Soochow University Robotics and Microsystems Control Center.

\section{References}

[1] Moghbel M, Mashohor S, Mahmud R, et al. Review of liver segmentation and computer assisted detection/diagnosis methods in computed tomography[J]. ARTIFICIAL INTELLIGENCE REVIEW,2018,50(4):497-537.

[2] Duncan JS, Ayache N. Medical image analysis: Progress over two decades and the challenges ahead[J]. IEEE transactions on pattern analysis and machine intelligence. 2000, Vol.22(1): 85-106.

[3] Dirk Smeets,Dirk Loeckx,Bert Stijnen,Bart De Dobbelaer,Dirk Vandermeulen,Paul Suetens. Semi-automatic level set segmentation of liver tumors combining a spiral-scanning technique with supervised fuzzy pixel classification[J]. Medical Image Analysis,2009,14(1).

[4] Bing Nan Li,Chee Kong Chui,Stephen Chang,Sim Heng Ong. A new unified level set method for semi-automatic liver tumor segmentation on contrast-enhanced CT images[J]. Expert Systems With Applications,2012,39(10).

[5] Yrjö Häme,Mika Pollari. Semi-automatic liver tumor segmentation with hidden Markov measure field model and non-parametric distribution estimation[J]. Medical Image Analysis,2012,16(1).

[6] Laurent Massoptier, Sergio Casciaro. A new fully automatic and robust algorithm for fast segmentation of liver tissue and tumors from CT scans[J].European Radiology, 2008, Vol.18 (8), pp.1658-1665

[7] Bo Liu,H.D. Cheng,Jianhua Huang,et al. Fully automatic and segmentation-robust classification of breast tumors 
based on local texture analysis of ultrasound images[J]. Pattern Recognition,2009,43(1).

[8] Min Xian,Yingtao Zhang,H.D. Cheng. Fully automatic segmentation of breast ultrasound images based on breast characteristics in space and frequency domains[J]. Pattern Recognition, 2015,48(2).

[9] Sangeethapriya R, Lakshmanan S, Senthilkumar J, et al. A Review on Medical Image Segmentation[J], 2018.

[10] Caselles V, Kimmel R, Sapiro G J I J O C V. Geodesic active contours[J], 1997, 22(1): 61-79.

[11] Kass M, Witk in A, Terzopoulos D.Snakes:Active contour models.International Journal of Computer Vision, 1987;1 (4) :321-331.

[12] Li C, Xu C, Gui C, et al. Distance Regularized Level Set Evolution and Its Application to Image Segmentation[J]. IEEE Transactions on Image Processing, 2010, 19(12): 3243-3254.

[13] ZHANG Minghui,Lu Zhentai,Zhang Juan,etc.Brain Image Segmentation Based on Multiple Atlas Active Contour Model[J]. Chinese Journal of Computers, 2016, 39 (7) :1490-1500.

[14] Wang L, Chang Y, Wang H, et al. An active contour model based on local fitted images for image segmentation[J]. Information Sciences, 2017, S418-419:61-73.

[15] WEI Chengcheng, YI Xuming. An Improved Method for Image Segmentation Based on DRLSE Level Set[J].Journal of Graphics,2019,40(05):885-891.

[16] CHAN T F,VESE L A.Active contour without edges[J].IEEE Transactionson Image Processing,2001,10(2):266-277.

[17] Li C, Kao C Y, Gore J C, et al. Minimization of Region-Scalable Fitting Energy for Image Segmentation[J], 2008, 17(10): 1940-1949.

[18] AKRAM F,GARCIA M A,PUIG D.Active contours driven by local and global fitted image models for image segmentation robust to intensity inhomogeneity[J].Plos One, 2017,12(4):e0174813.

[19] ZHANG Kaihua,ZHANG Lei,LAM K M,et al.A level set approach to image segmentation with intensity inhomogeneity[J].IEEE Transactions on Cybernetics,2017,46(2):546-557.

[20] ZHAO Wencheng,XU Xianze,ZHU Yanyan,et al.Active contour model based on local and global Gaussian fitting energy for medical image segmentation[J].Optik-International Journal for Light and Electron Optics,2018,158:1160-1169.

[21] SOOMRO S,MUNIR A,CHOI K N.Hybrid two-stage active contour method with region and edge informatio for intensity inhomogeneous image segmentation[J].Plos One,2018,13(1):e0191827.

[22] Lecun Y, Boser B, Denker J S, et al. Backpropagation applied to handwritten zip code recognition[J]. Neural Computation, 1989, 1(4):541-551.
[23] Greenspan H,Van Ginneken B,Summers RM.Guest editorial deep learning in medical imaging: Overview and future promise of an exciting new technique[J]. IEEE Transactions on Medical Imaging,2016,35(5):1153-1159.

[24] Lee J-G,Jun S,Cho Y-W,et al.Deep learning in medical imaging: general overview[J].Korean J Radiol,2017,18(4):570-584.

[25] Ben-Cohen A , Diamant I , Klang E, et al. Fully convolutional network for liver segmentation and lesions detection[C]. International Workshop on Large-Scale Annontation of Biomedical Data and Expert Label Synthesis, Athens, Greece, 2016,77-85.

[26] Ronneberger O, Fischer P, Brox T. U-Net: convolutional networks for biomedical image segmentation[C]// International International Conferenceon Medical image computing and computer-assisted intervention. Springer, Cham, 2015: 234-241.

[27] Suzuki Kenji,Huynh Hieu Trung,Liu Yipeng,etal. Computerized segmentation of liver in hepatic CT and MRI by means of level-set geodesic active contouring.[J]. Conference proceedings : ... Annual International Conference of the IEEE Engineering in Medicine and Biology Society. IEEE Engineering in Medicine and Biology Society. Annual Conference,2013,2013.

[28] Amir H. FORUZAN, Yen-Wei CHEN,etal. Segmentation of Liver in Low-Contrast Images Using K-Means Clustering and Geodesic Active Contour Algorithms[J]. The Institute of Electronics, Information and Communication Engineers,2013,E96.D(4).

[29] Suzuki Kenji,Kohlbrenner Ryan,Epstein Mark L,etal. Computer-aided measurement of liver volumes in CT by means of geodesic active contour segmentation coupled with level-set algorithms.[J]. Medical physics,2010,37(5).

[30] ZHAO Yannan,ZENG Yezhan. Liver tumor segmentation of abdominal CT image based on improved CV model[J]. China Computer \& Communication,2016(23):123-126.

[31] XIE Zhinan,ZHEN Dong,CHEN Jianyao,etal. A Tumor Segmentation Method of Improve Chan-Vese Model for Liver Cancer Ablation Computed Tomography Image.[J] Laser \& Optoelectronics Progress. 2017,54(02):240-245.

[32] Osher S, Fedkiw R, Piechor K. Level Set Methods and Dynamic Implicit Surfaces[J], 2004, 57(3): xiv+273.

\section{Figure Legends}

[1] Fig.1 Flow chart of image segmentation

[2] Fig.2 Segmentation of liver tumors (Category A)

[3] Fig.3 Segmentation of liver tumors (Category B)

[4] Fig.4 Segmentation of liver tumors (Category C)

[5] Fig.5 Energy functional curve (Category A,B,C)

[6] Fig.6 Surface of level set (Category A,B,C)

[7] Fig.7 Segmentation of liver nodules

\section{Table Legends}

[1] Table 1 Evaluation index 
Figures

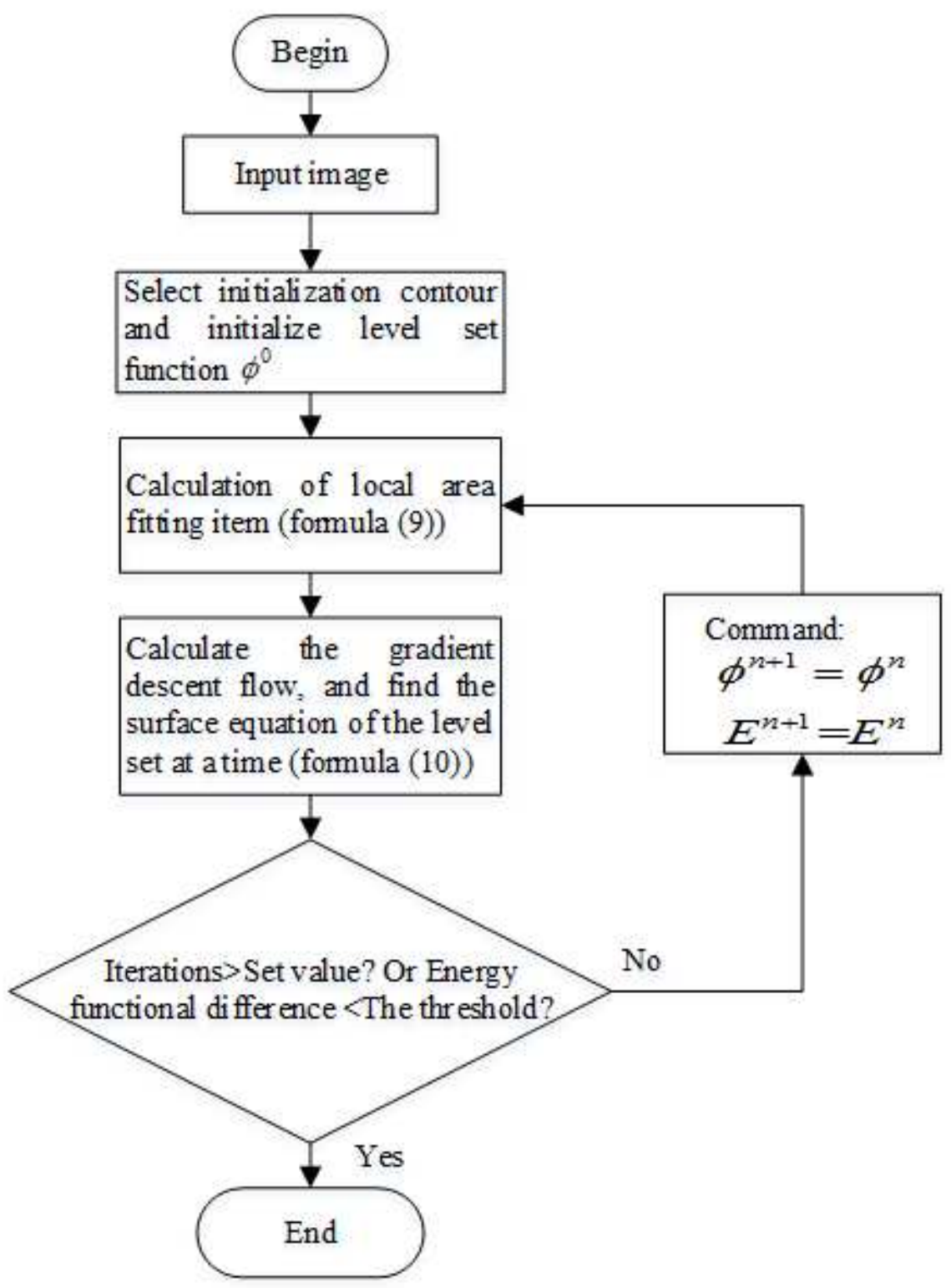

\section{Figure 1}

Flow chart of image segmentation 


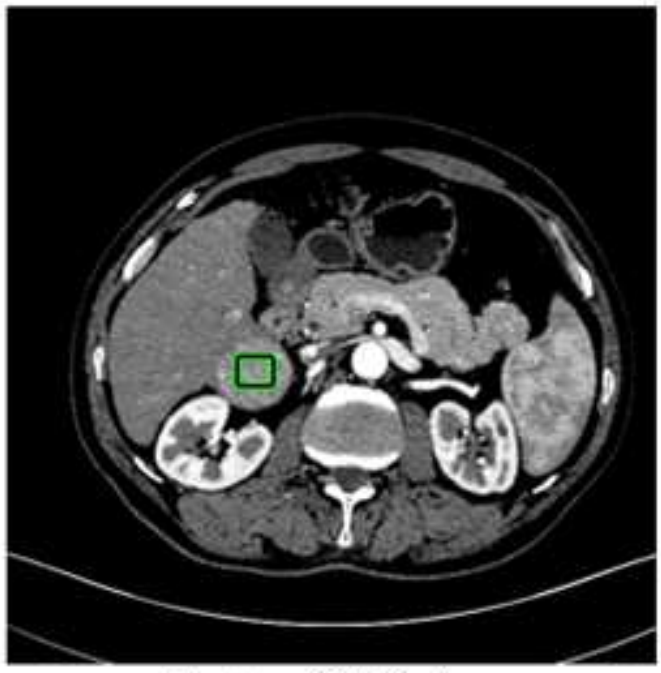

Contour initialization

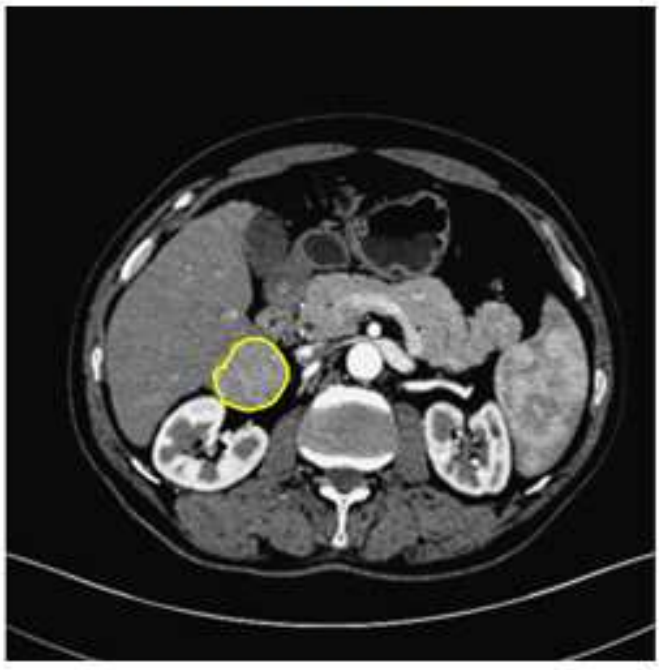

RSF

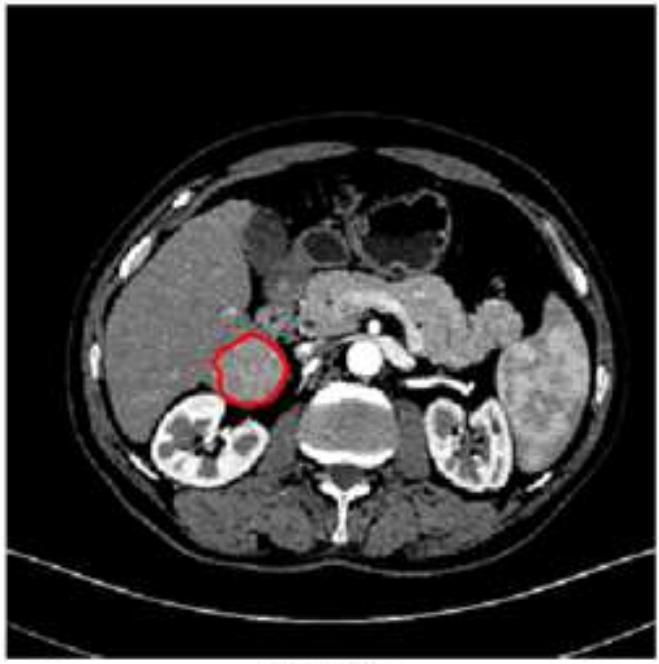

DRLSE

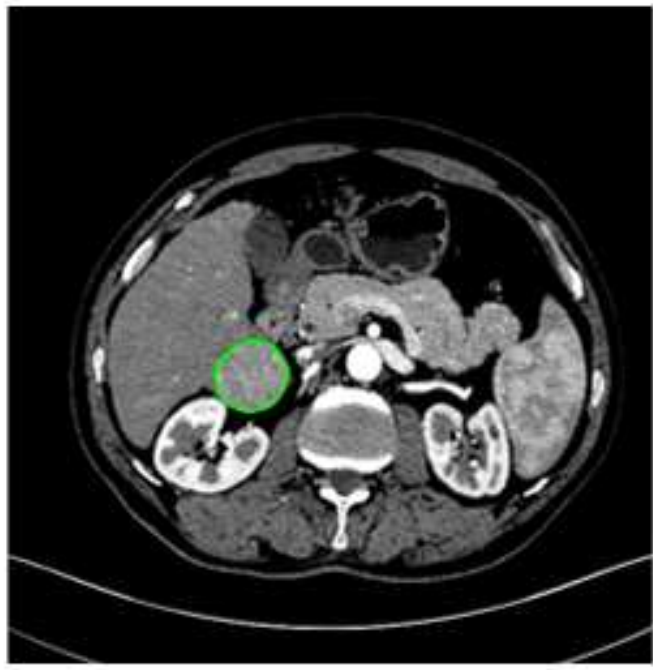

The model of this paper

Figure 2

Segmentation of liver tumors (Category A) 


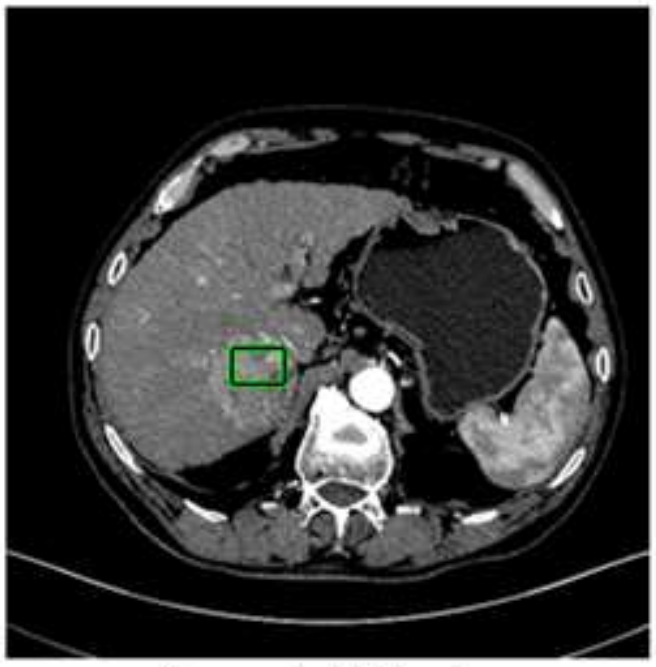

Contour in itialization

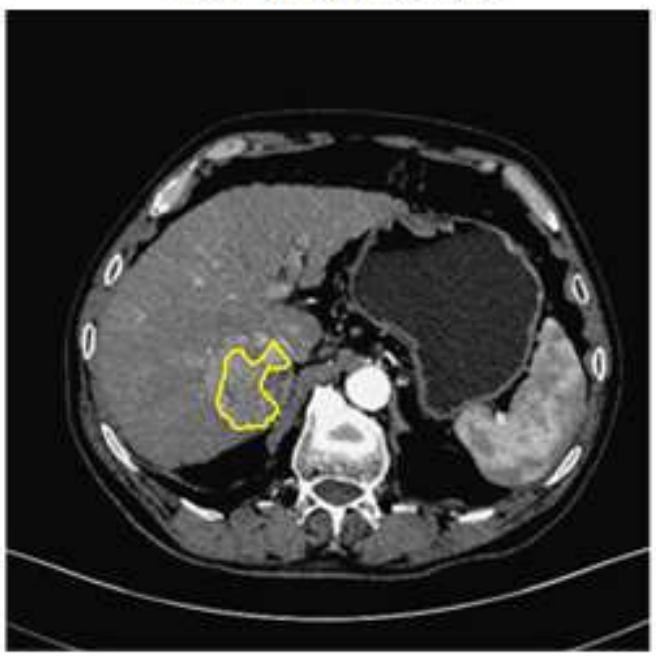

RSF

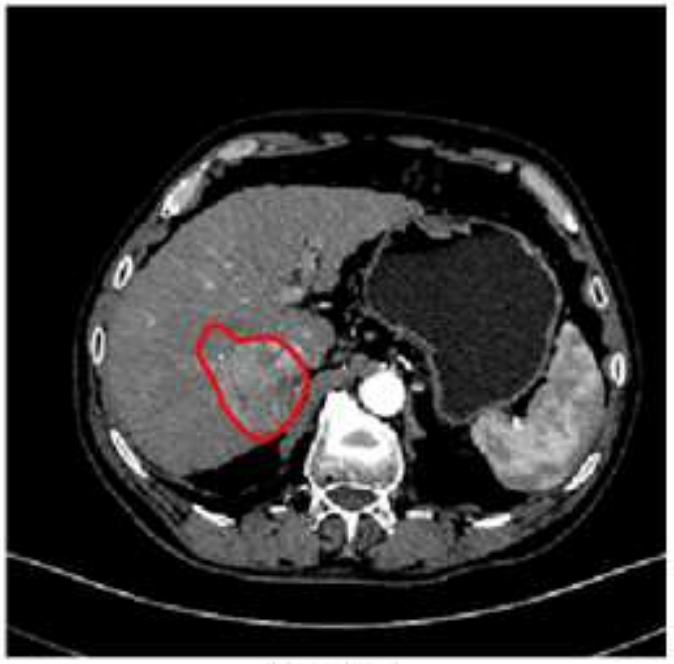

DRLSE

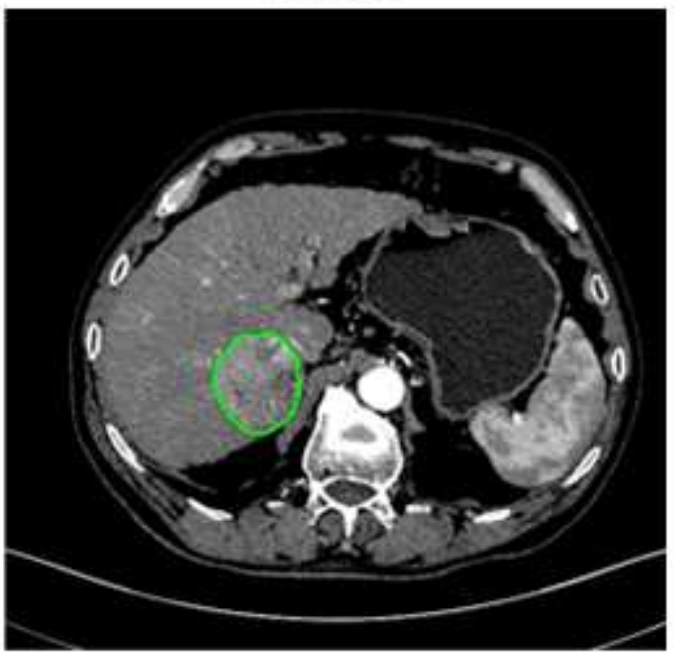

The model of this paper

\section{Figure 3}

Segmentation of liver tumors (Category B) 


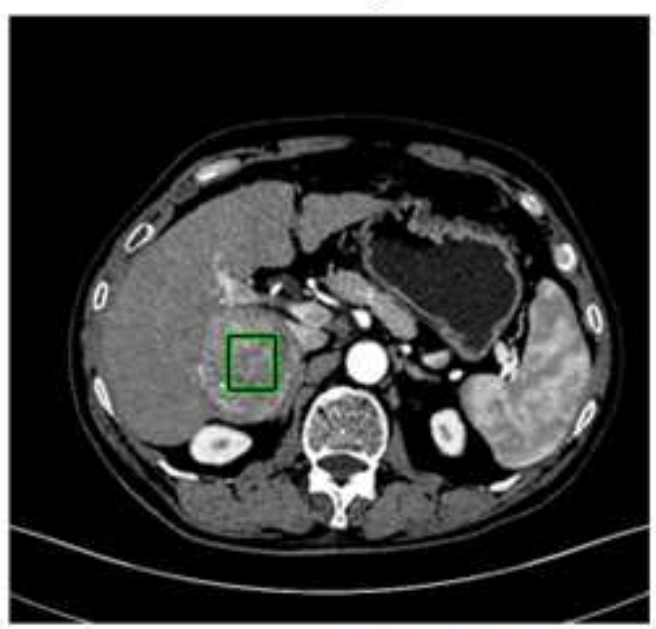

Con to ur initialization

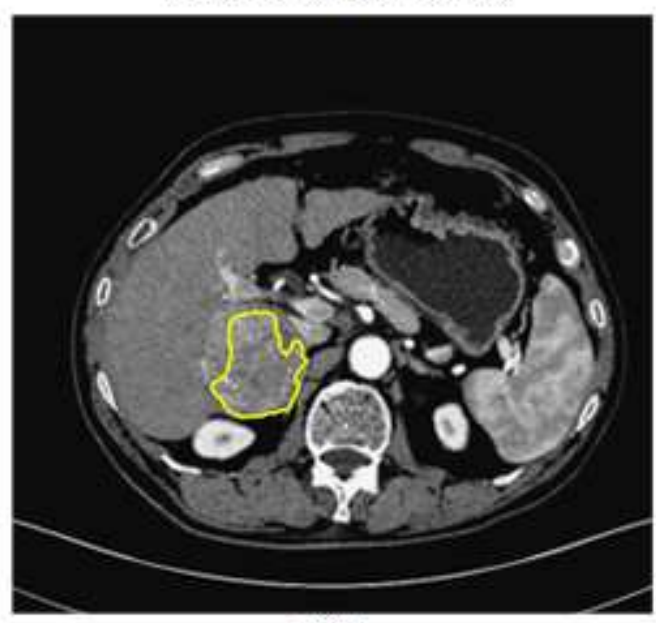

RSF

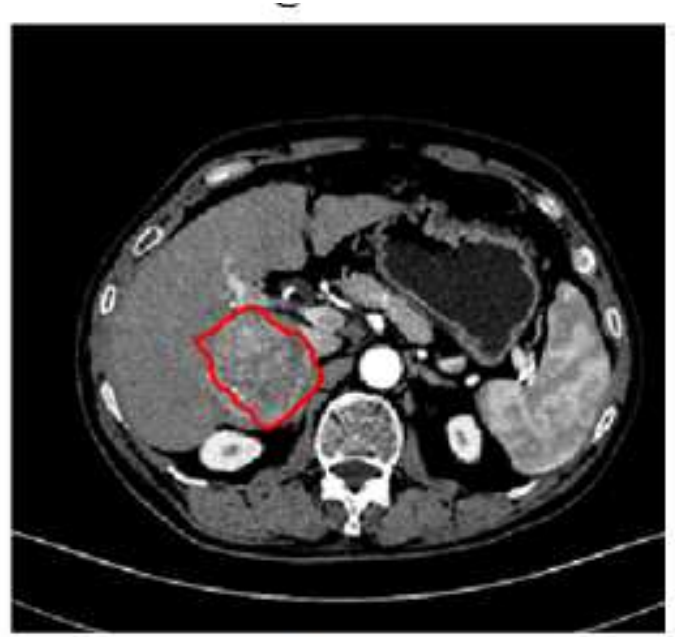

DRLSE

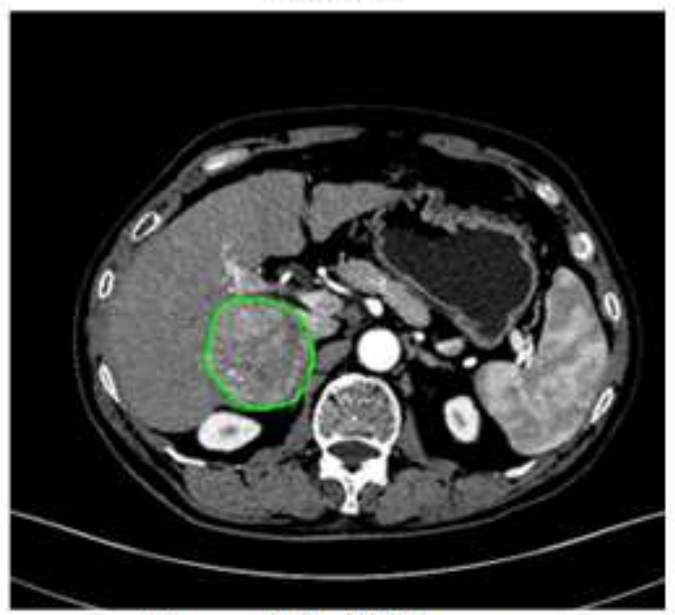

The model of this p aper

Figure 4

Segmentation of liver tumors (Category C) 

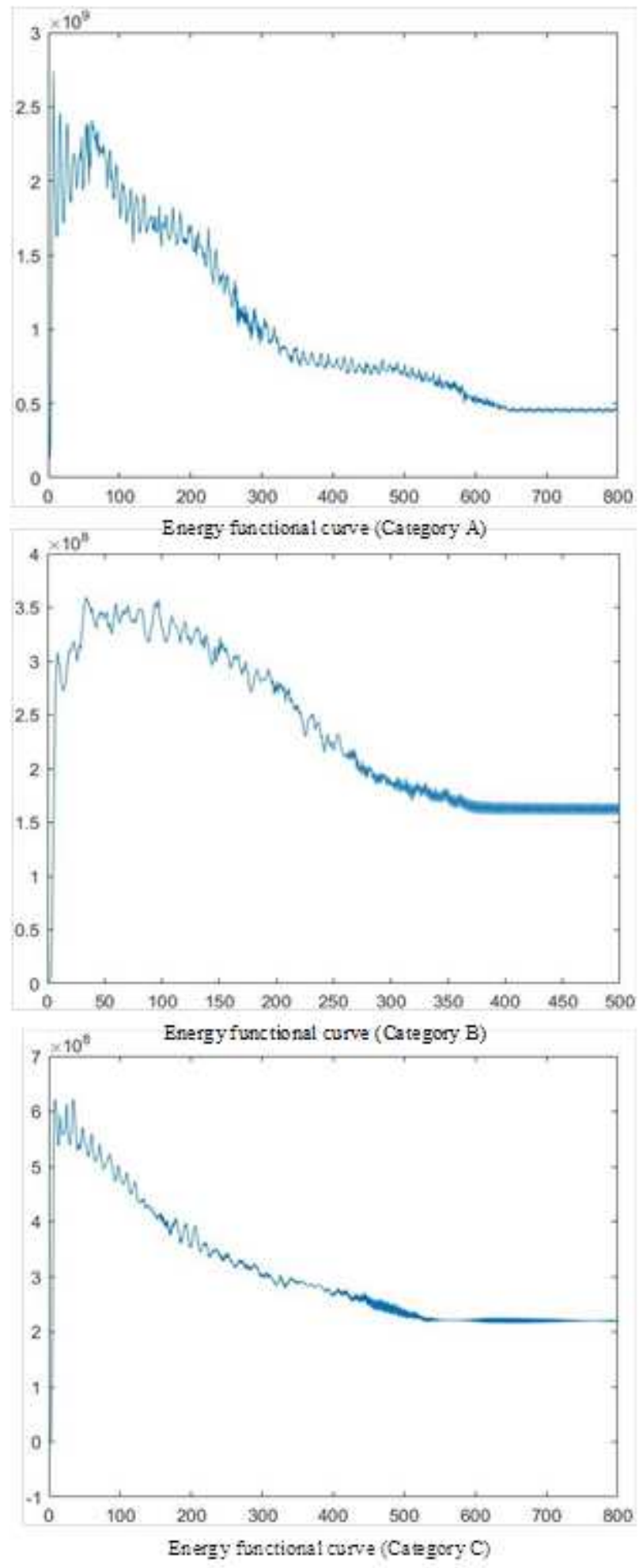

Figure 5

Energy functional curve (Category $A, B, C$ ) 


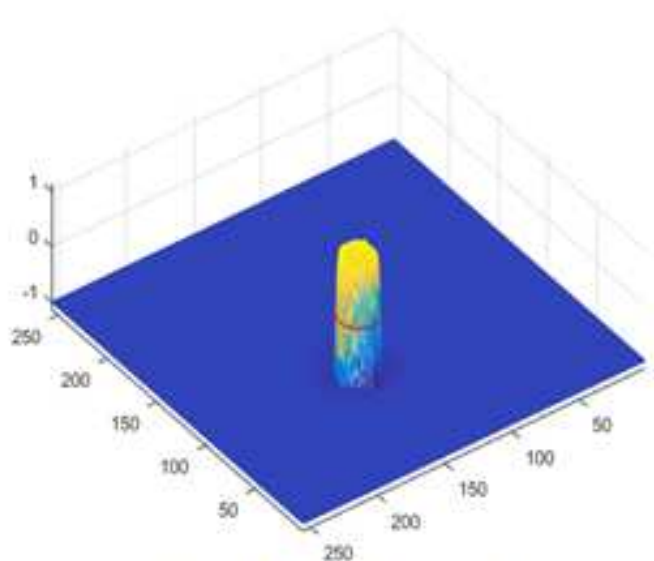

Sufife of tevel set (Caegry A)

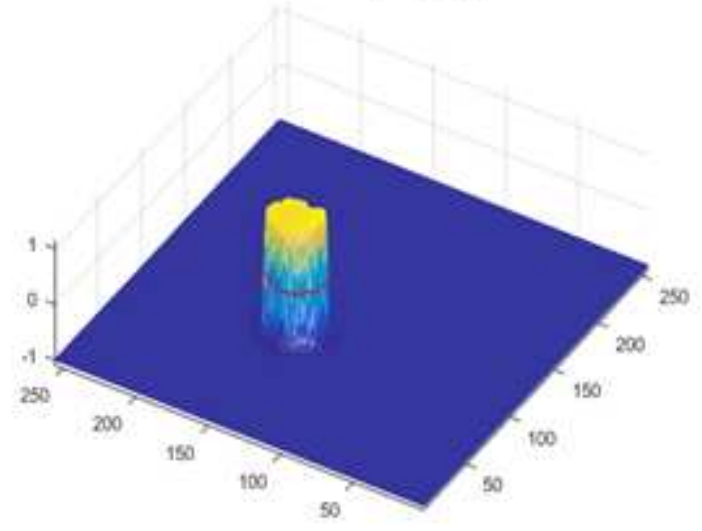

Surface of level set(CatgoryB)

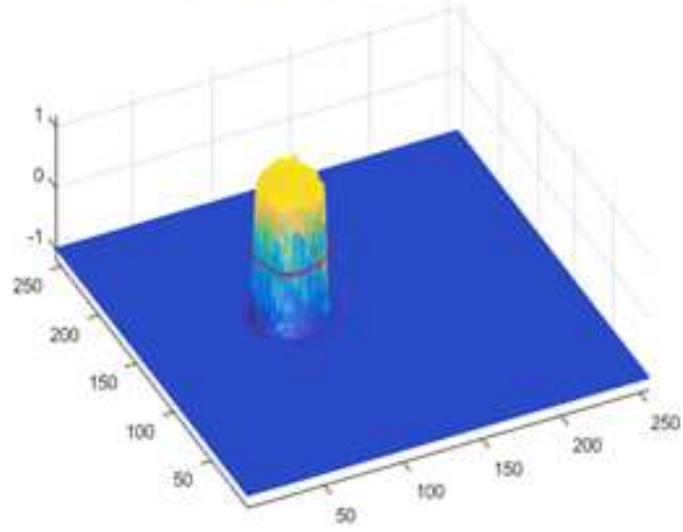

Surface of level ser(CategoryC)

Figure 6

Surface of level set (Category $A, B, C$ ) 


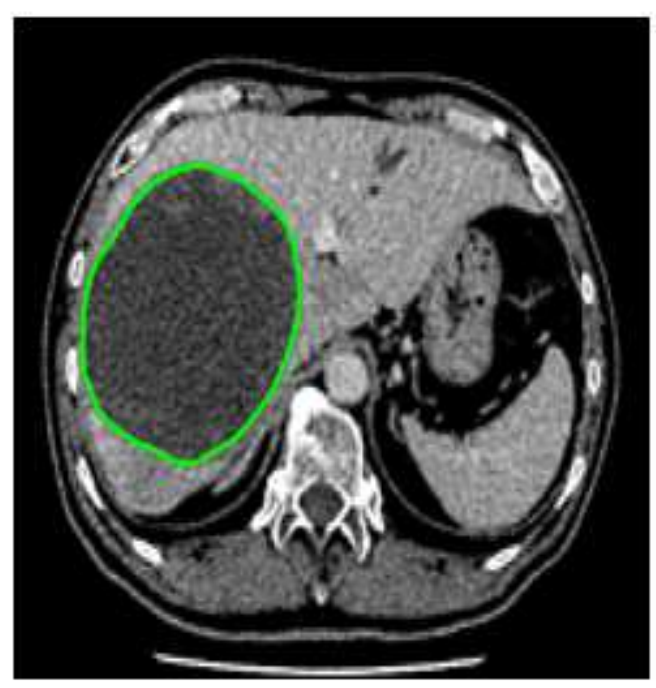

a

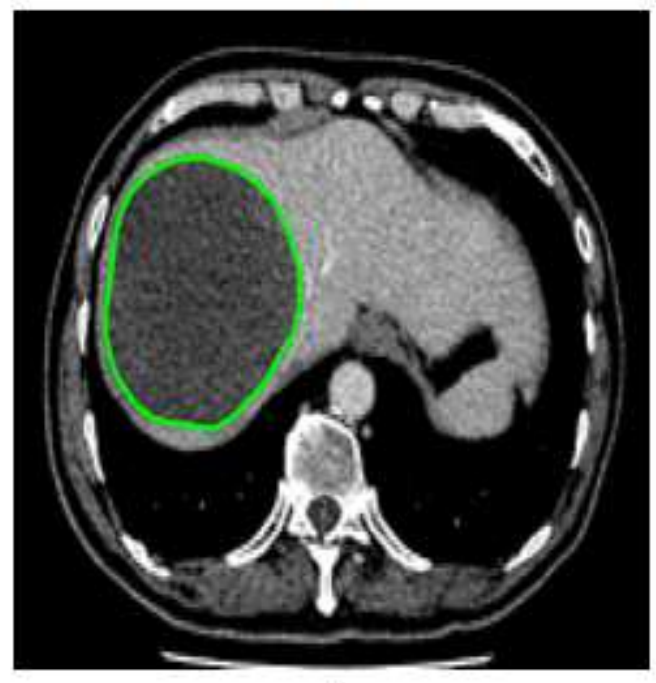

c

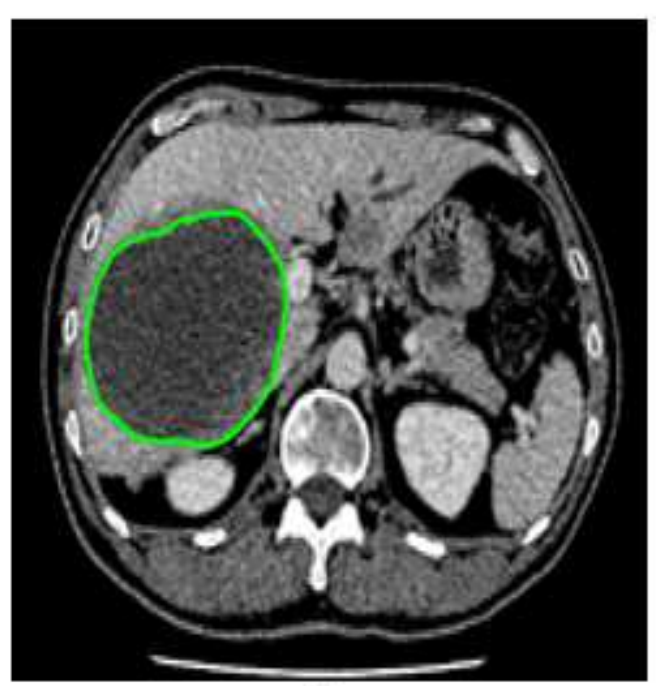

b

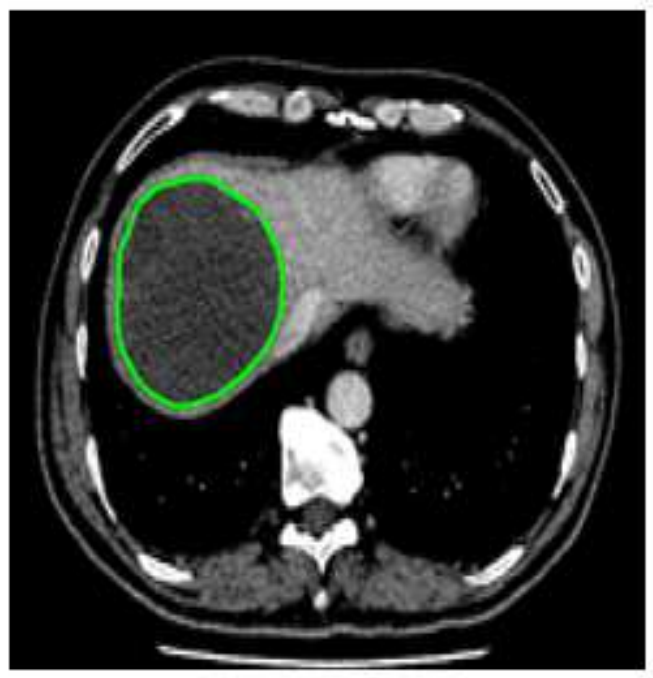

d

Figure 7

Segmentation of liver nodules 